\title{
Concurrent electromigration and creep in lead-free solder
}

\section{Citation}

Pharr, Matt, Kejie Zhao, Zhigang Suo, Fan-Yi Ouyang, and Pilin Liu. 2011. “Concurrent Electromigration and Creep in Lead-Free Solder." Journal of Applied Physics 110 (8): 83716. https://doi.org/10.1063/1.3656002.

\section{Permanent link}

http://nrs.harvard.edu/urn-3:HUL.InstRepos:41461226

\section{Terms of Use}

This article was downloaded from Harvard University's DASH repository, and is made available under the terms and conditions applicable to Other Posted Material, as set forth at http:// nrs.harvard.edu/urn-3:HUL.InstRepos:dash.current.terms-of-use\#LAA

\section{Share Your Story}

The Harvard community has made this article openly available.

Please share how this access benefits you. Submit a story.

Accessibility 


\title{
Concurrent electromigration and creep in lead-free solder
}

\author{
Matt Pharr, ${ }^{1}$ Kejie Zhao, ${ }^{1}$ Zhigang Suo, ${ }^{1, a)}$ Fan-Yi Ouyang, ${ }^{2,3}$ and Pilin Liu ${ }^{2}$ \\ ${ }^{1}$ School of Engineering and Applied Sciences and Kavli Institute, Harvard University, Cambridge, \\ Massachusetts 02138, USA \\ ${ }^{2}$ Intel Corporation, Assembly Technology Development Center, Chandler, Arizona 85226, USA \\ ${ }^{3}$ Department of Engineering and System Science, National Tsing Hua University, Hsinchu 300, Taiwan
}

(Received 18 July 2011; accepted 13 September 2011; published online 28 October 2011)

\begin{abstract}
When electric current flows in a solder bump, electromigration generates stress, but creep relaxes it. After some time, the bump develops a steady-state stress field. We present a theory to show that the two processes - electromigration and creep — set an intrinsic length. When the intrinsic length is large compared to the height of the bump, electromigration is fast relative to creep and the steady-state stress field is linearly distributed in the bump. When the intrinsic length is small compared to the height of the bump, electromigration is slow relative to creep and the steady-state stress field nearly vanishes in the bump, except in a thin layer along the boundary of the bump. We further show that a critical electric current exists, below which the bump can sustain the steady-state stress field without forming voids. Theoretical predictions are compared with existing experimental observations. (C) 2011 American Institute of Physics. [doi:10.1063/1.3656002]
\end{abstract}

\section{INTRODUCTION}

In microelectronic devices, intense electric currents motivate atoms to diffuse - a process known as electromigration. Although electromigration is present in many components of the devices, the current density needed to cause electromigration in solder bumps is much smaller than in any other components. ${ }^{1}$ Electromigration can cause the bumps to form voids, leading to failure. ${ }^{2}$

Blech observed, in an interconnect line, a critical current density, below which electromigration does not cause damage. ${ }^{3}$ This observation was interpreted by Blech and Herring as follows: ${ }^{4}$ As the electric current causes atoms to migrate from one end to the other along the interconnect, a gradient of stress builds up, counteracting electromigration. After some time, the interconnect develops a steady-state stress gradient and net migration of atoms stops. The magnitude of the stress at either end of the interconnect is limited by some mechanisms of failure, such as the formation of voids and extrusion of the metal into the surrounding dielectrics, so that the achievable stress gradient is large in short interconnects. Consequently, short interconnects are immortal, immune from electromigration-induced failure. ${ }^{5-8}$ This consideration has played a significant role in the design of interconnects. $^{1,9-11}$

It is tempting to apply the Blech-Herring analysis to solder bumps. However, solder bumps require an additional consideration. During operation, a bump can reach $75 \%$ of its melting point ${ }^{12}$ and the bump creeps. ${ }^{13}$ The bump is often surrounded by relatively compliant molding compounds, possibly allowing creep to relax the stress in the bump. By contrast, an interconnect is often confined by relatively stiff dielectrics, which enables the interconnect to retain hydrostatic stress in the presence of creep. ${ }^{14}$

\footnotetext{
${ }^{\text {a) }}$ Author to whom correspondence should be addressed. Electronic mail: suo@seas.harvard.edu.
}

To apply the Blech-Herring analysis to solder bumps, this paper considers concurrent electromigration and creep. When electric current flows in the bump, stress is generated by electromigration, but relaxed by creep. After some time, the bump develops a steady-state stress field. To determine this steadystate stress field, Sec. II reviews a theory of concurrent electromigration and creep. Section III shows that the relative rates of the two processes - electromigration and creep can be quantified by an intrinsic length. When the height of the bump is small compared to the intrinsic length, electromigration is fast relative to creep and the theory recovers the classical prediction of Blech and Herring, in which the steady-state stress is linearly distributed in the bump. When the height of the bump is large compared to the intrinsic length, electromigration is slow relative to creep and the theory reveals a unique situation, in which the steady-state stress nearly vanishes in the bump, except in a thin layer around the boundary of the bump.

These two limiting cases, as well as the intermediate behavior, are illustrated in Sec. IV with a thin film. Section $\mathrm{V}$ then establishes a critical stress, above which voids will grow, based on the Laplace condition. Section VI uses Pbfree solder data to investigate how the critical current density needed to cause void nucleation depends on various parameters of the solder. We find that, at normal operating temperatures, for a $\mathrm{SnAg}_{4} \mathrm{Cu}_{0.5}$ solder bump larger than $\sim 1 \mu \mathrm{m}$, creep is important and the Blech-Herring analysis is inadequate. Section VII compares the theoretical predictions to experimental observations. Finally, in Sec. VIII, we justify our approximation of the solder bump as a thin film by comparison to $3 \mathrm{D}$ simulations.

\section{THEORY OF CONCURRENT ELECTROMIGRATION AND CREEP}

When electric current passes through a solder bump, the flow of electrons - the electron wind - motivates atoms of 
the bump to diffuse. As atoms relocate from the cathode to the anode, a field of stress builds up in the bump, tensile at the cathode, and compressive at the anode. While electromigration generates stress, creep tends to relax it. After some time, the concurrent electromigration and creep set up a steadystate stress field in the bump. This section summarizes a previously developed theory of concurrent creep and diffusion. ${ }^{15}$

Following Darken, ${ }^{16}$ we imagine that inert markers are scattered throughout a material. These markers do not diffuse; rather, they move along with the deformation of the material. It should be noted that this idea is not merely hypothetical. Movements of markers in solder bumps have been visualized with diamond particles and arrays of nanoindentations. ${ }^{17,18}$ Let $\left(x_{1}, x_{2}, x_{3}\right)$ represent the coordinates of a fixed space, and $v_{i}\left(x_{1}, x_{2}, x_{3}, t\right)$ be the velocity vector of the marker at position $\left(x_{1}, x_{2}, x_{3}\right)$ at time $t$. The gradient of the marker velocity defines the strain-rate tensor

$$
d_{i j}=\frac{1}{2}\left(v_{i, j}+v_{j, i}\right) .
$$

Let $\Omega$ be the volume per atom in the body. Imagine a plane fixed in space and perpendicular to the axis $x_{i}$. The net atomic flux, $N_{i}$, is the number of atoms that move across the plane per unit area and per unit time, as shown in Fig. 1. We can independently measure this net atomic flux and the marker velocity. The convection flux, $v_{i} / \Omega$, is the number of atoms moving with the marker across the plane per unit area per unit time. The difference between the two fluxes defines the self-diffusion flux $J_{i}$, namely,

$$
N_{i}=J_{i}+\frac{v_{i}}{\Omega} .
$$

This equation states that the net flux, $N_{i}$, is the sum of the diffusion flux, $J_{i}$, and the convection flux, $v_{i} / \Omega$.

To emphasize the main features of this theory with minimum complication, we neglect elasticity. We also know that abundant sources and sinks, such as grain boundaries and dislocations, exist in a solder bump, as illustrated in Fig. 2. As noted by Balluffi, these sources and sinks tend to maintain equilibrium concentrations of vacancies, which are usually small. ${ }^{19}$ Therefore, a fixed volume will contain a constant number of atoms at all time and the net atomic flux is divergence free,

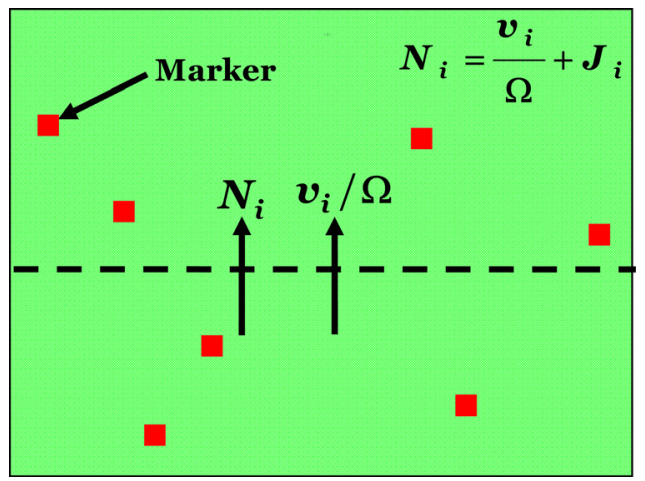

FIG. 1. (Color online) The marker velocity determines the convection flux, $v_{i} / \Omega$. The net atomic flux $N_{i}$ can also be independently measured. The atomic flux in excess of the convective flux defines the diffusion flux $J_{i}$. (a)

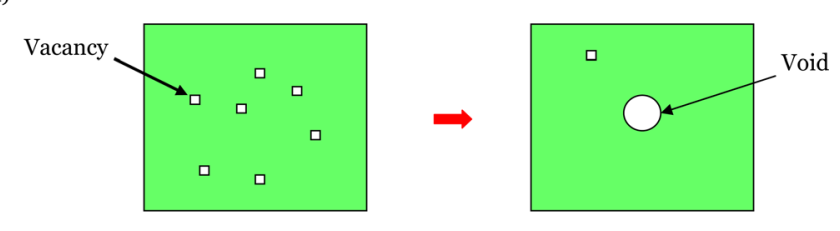

(b)

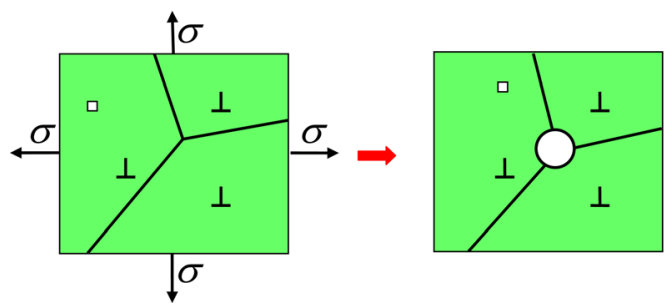

FIG. 2. (Color online) Two possible scenarios exist for growth of a void. In (a), flux divergence generates vacancies, which coalesce to form voids. In (b), sinks/sources keep vacancy concentrations at the equilibrium level. Flux divergence generates stress under constraint, leading to growth of a void.

$$
N_{k, k}=0 .
$$

A combination of Eqs. (2) and (3) gives

$$
v_{k, k}=-\Omega J_{k, k} .
$$

This equation states that, even though the material is incompressible, the marker velocity has a divergence to compensate for the divergence in the diffusion flux.

We take the strain-rate to be the sum of that due to creep, $d_{i j}^{C}$, and that due to diffusion, $d_{i j}^{D}$,

$$
d_{i j}=d_{i j}^{C}+d_{i j}^{D} .
$$

We assume that the divergence in diffusion flux causes an equal strain rate in all three directions,

$$
d_{i j}^{D}=-\frac{\Omega}{3} J_{k, k} \delta_{i j},
$$

where $\delta_{i j}=1$ when $i=j$ and $\delta_{i j}=0$ otherwise. We adopt this rule based on experimental evidence of its validity: it is consistent with observations of lateral shrinkage of thin foils during selective evaporation, ${ }^{20}$ and it correctly predicts the bending of a thin foil diffusion couple. ${ }^{21}$ However, it can be modified if, for some reason, atoms are preferentially placed on certain crystal planes. ${ }^{22}$

Combining Eqs. (1) and (4)-(6) gives the creep strain rate in terms of the marker velocity field,

$$
d_{i j}^{C}=\frac{1}{2}\left(v_{i, j}+v_{j, i}\right)-\frac{1}{3} v_{k, k} \delta_{i j} .
$$

Since creep generates negligible acceleration, the force balance equations can be written as

$$
\begin{aligned}
& \left(\sigma_{i j}\right)_{, j}=0, \text { in the volume, } \\
& \sigma_{i j} n_{j}=t_{i}, \text { on the surface. }
\end{aligned}
$$

The mean stress is defined as 


$$
\sigma_{m}=\frac{1}{3}\left(\sigma_{11}+\sigma_{22}+\sigma_{33}\right)
$$

The chemical potential induced by this mean stress is $\mu=-\Omega \sigma_{m}$. This quantity represents the free energy change associated with moving an atom in a stress-free reference body to a point in the material subject to mean stress $\sigma_{m}$. Following Blech and Herring, ${ }^{4}$ both the electron wind force and the gradient of the chemical potential drive diffusion,

$$
J_{i}=\frac{D}{\Omega k T}\left[F_{i}+\left(\Omega \sigma_{m}\right)_{i}\right],
$$

where $D$ is the self-diffusion coefficient, $k T$ is the temperature in the unit of energy, and $F_{i}$ is the electron wind force. The electron wind force relates to the electric current through the relation $F_{i}=Z e \rho j_{i}$, where $Z$ is the effective valence of atoms, $e$ is the elementary charge, $\rho$ is the resistivity, and $j$ is the current density. ${ }^{23}$

The deviatoric stress tensor is given by

$$
s_{i j}=\sigma_{i j}-\sigma_{m} \delta_{i j} \text {. }
$$

The equivalent stress is defined as

$$
\sigma_{e}=\sqrt{3 s_{i j} s_{i j} / 2}
$$

The creep strain rate is commonly written as the product of the deviatoric stress and some function of the equivalent stress

$$
d_{i j}^{C}=\alpha\left(\sigma_{e}\right) s_{i j}
$$

The function $\alpha\left(\sigma_{\mathrm{e}}\right)$ is determined by fitting the relation between stress and strain rate measured under a simple stress state. For instance, performing a uniaxial tension test, experimentalists may obtain a constitutive equation with the general form

$$
\frac{d_{11}^{C}}{\dot{\varepsilon}_{0}}=f\left(\frac{\sigma_{11}}{\sigma_{0}}\right),
$$

where $d_{11}^{C}$ and $\sigma_{11}$ are the strain rate and stress in the loading direction, $\sigma_{0}$ is a reference stress, and $\dot{\varepsilon}_{0}$ is the strain rate in the loading direction at the stress $\sigma_{0}$. For a uniaxial tensile test, the equivalent stress is $\sigma_{e}=\sigma_{11}$ and the deviatoric stress is $s_{11}=2 \sigma_{11} / 3$. Thus, equating Eqs. (14) and (15) gives $\alpha\left(\sigma_{e}\right)=3 \dot{\varepsilon}_{0} f\left(\sigma_{e} / \sigma_{0}\right) /\left(2 \sigma_{e}\right)$ and the general 3D constitutive equation is

$$
\frac{d_{i j}^{C}}{\dot{\varepsilon}_{0}}=\frac{3}{2} \frac{s_{i j}}{\sigma_{e}} f\left(\frac{\sigma_{e}}{\sigma_{0}}\right) .
$$

Define the effective creep strain rate as $d_{e}^{C}=\left(2 d_{i j}^{C} d_{i j}^{C} / 3\right)^{1 / 2}$. This definition, in combination with Eqs. (12), (13), and (16), gives that $d_{e}^{C} / \dot{\varepsilon}_{0}=f\left(\sigma_{e} / \sigma_{0}\right)$. Thus, the function relates the effective creep strain rate to the effective stress.

\section{SCALING ANALYSIS AND LIMITING CASES}

This theory has a characteristic length, as can be seen through a scaling analysis. Inserting the creep model in
Eq. (16) and the creep strain rate expression (7) into the force balance in Eq. (8), we obtain

$$
\left[\frac{1}{3} \frac{\sigma_{e}}{\dot{\varepsilon}_{0} f\left(\sigma_{e} / \sigma_{0}\right)}\left(v_{i, j}+v_{j, i}-\frac{2}{3} v_{k, k} \delta_{i j}\right)\right]_{, j}+\left(\sigma_{m}\right)_{, i}=0 .
$$

Inserting the diffusion law in Eq. (11) into the kinematic constraint in Eq. (4), we obtain

$$
v_{k, k}=-\left\{\frac{D}{k T}\left[F_{k}+\left(\Omega \sigma_{m}\right)_{, k}\right]\right\}_{, k} .
$$

As mentioned above, the effective stress $\sigma_{e}$ is related to the effective strain rate $d_{e}^{C}$ through the function $f$. The effective strain rate is defined as $d_{e}^{C}=\left(2 d_{i j}^{C} d_{i j}^{C} / 3\right)^{1 / 2}$, and the creep strain rate tensor $d_{i j}^{C}$ is related to the velocity field by Eq. (7). Consequently, Eqs. (17) and (18) consist of four partial differential equations that govern the four fields $v_{1}, v_{2}, v_{3}$, and $\sigma_{m}$. Under the special condition that the diffusion flux is divergence-free, $J_{k, k}=0$ and $v_{k, k}=0$, Eq. (17) recovers Stokes's equation for creep and Eq. (18) recovers Herring's equation for self-diffusion.

Let $\Lambda$ be the length to be determined. Scale the stress by $\sigma_{0}$, the marker velocities by $\Lambda \dot{\varepsilon}_{0}$, the wind forces by $\Omega \sigma_{0} / \Lambda$, and the spatial coordinates by $\Lambda$. Equations (17) and (18) become dimensionless and parameter-free, provided we set

$$
\Lambda=\sqrt{\frac{D \Omega \sigma_{0}}{k T \dot{\varepsilon}_{0}}} .
$$

This length characterizes the relative rate of creep and diffusion. Let $H$ be a length scale in the boundary-value problem, e.g., the height of a solder bump. Large values of $\Lambda / H$ indicate that the time necessary for diffusion across the material is fast relative to the time necessary for creep.

We now wish to investigate how $\Lambda / H$ affects the steady-state stress field in a solder bump. Since the electron wind force is roughly constant through the thickness of the solder, it alone does not result in a divergence in diffusion flux. However, different materials contact the solder at its boundaries and atoms diffuse at different rates on each side of the boundary. For instance, the diffusivity of $\mathrm{Sn}$ in $\mathrm{Sn}$ is much faster than that of $\mathrm{Sn}$ in $\mathrm{Cu}$. This results in a divergence in the diffusion flux of Sn at the boundary between $\mathrm{Sn}$ and $\mathrm{Cu}$. For a steady state to be reached, a stress gradient must be built up to counteract this divergence in diffusion flux. The length over which this stress gradient will be built up depends on the ratio $\Lambda / H$.

In one limit, $\Lambda / H \gg 1$, diffusion is so much faster than creep that the effect of creep can be neglected. In this limit, the theory outlined in Sec. II will reduce to the analysis of Blech and Herring. ${ }^{4}$ For a steady state to be reached, the diffusion flux must vanish, $J_{i}=0$. For a $1 \mathrm{D}$ wind force in the $x_{3}$ direction, Eq. (11) then gives $F_{3}+\Omega\left(d \sigma_{m} / d x_{3}\right)=0$ in the steady state. The stress gradient is a constant governed by the boundary conditions and is built up through the entire thickness of the solder. 
In the other limit, $\Lambda / H \ll 1$, creep is extremely fast relative to diffusion. Since creep tends to relax stress, in this limit, it seems that the stress may be zero everywhere in the solder. However, as previously mentioned, there is a flux divergence at the boundary. To satisfy this boundary condition, a stress gradient must be built up to offset this flux divergence. Since the characteristic length $\Lambda$ is small relative to the size $H$ of the boundary value problem, this stress will be localized near the boundary of the solder, as illustrated in Fig. 3.

\section{STRESS IN A THIN FILM}

To see how the physical ideas developed in Sec. III arise mathematically, let us consider a thin film of a solder material sandwiched between two other materials, as illustrated in Fig. 4. Let us also assume the material under uniaxial tension obeys power-law creep $\dot{\varepsilon}=\dot{\varepsilon}_{0}\left(\sigma / \sigma_{0}\right)^{n}$. An electron wind force will cause atoms to diffuse in the direction of the wind force, creating a state of compression near the anode and tension near the cathode. This stress state is biaxial with $\sigma_{11}=\sigma_{22}$, and the equivalent stress is $\sigma_{e}=\left|\sigma_{11}\right|$.

Equation (11) gives the diffusion flux as $J_{3}=(D / \Omega k T)$ $\left(F_{3}+[2 \Omega / 3] d \sigma_{11} / d x_{3}\right)$. This diffusion flux induces a strain rate $d_{11}^{D}=-(\Omega / 3) d J_{3} / d x_{3}$. The bounding materials above and below the film constrain it from deforming laterally, so that the total strain rates vanish in the lateral directions: $d_{11}=0$. Scaling the stresses by $\sigma_{0}$ and the spatial coordinates by the thickness $H$, we obtain

$$
-\frac{4}{9}\left(\frac{\Lambda}{H}\right)^{2} \frac{d^{2} \tilde{\sigma}_{11}}{d \tilde{x}_{3}^{2}}+\tilde{\sigma}_{11}^{n}=0
$$

where $\Lambda$ is the characteristic length given in Eq. (19) and the tildes represent non-dimensional quantities. We have dropped the absolute value by examining this expression on the domain $-1 / 2 \leq \tilde{x}_{3} \leq 0$, where the material is under tension.

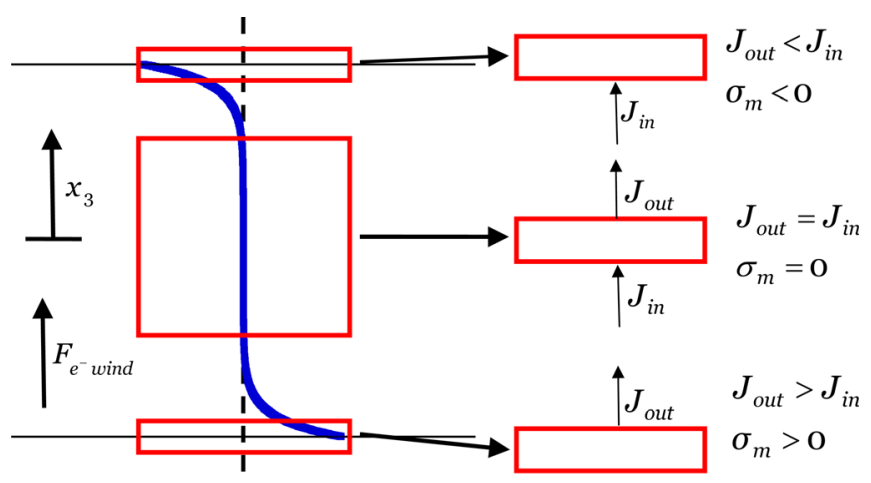

FIG. 3. (Color online) Solder bump with stress distribution for a rapidly creeping material. The horizontal axis is the stress level and the vertical axis is the position through the thickness of the solder bump. The solid line shows the stress distribution through the thickness of the solder $\sigma_{m}\left(x_{3}\right)$. The dotted line indicates zero stress; to the left of this curve, the material is in compression, and to the right of the curve, the material is in tension. Stress is nearly zero everywhere, but deviates from zero near the boundaries to counteract the flux divergence there. The flux divergence is illustrated in the three boxes, which represent differential volume elements at three locations through the thickness.

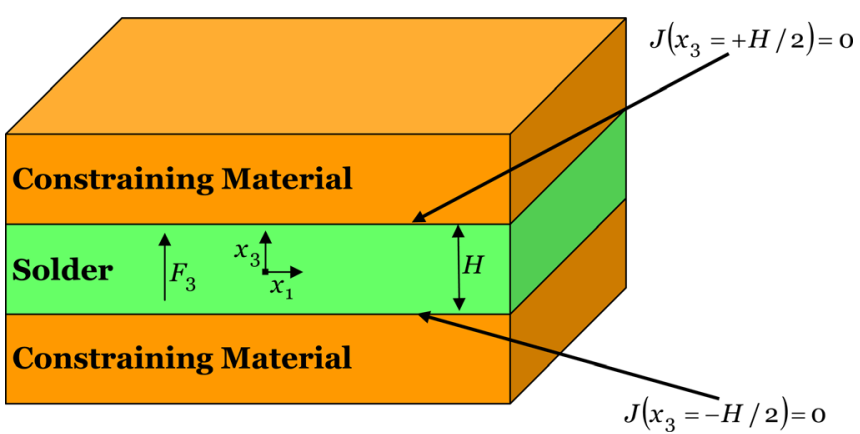

FIG. 4. (Color online) A thin film of a solder material, height $H$, is subject to a through-thickness electron wind force, $F_{3}$. The film is sandwiched between two materials that constrain it. Atoms do not diffuse into the two surrounding materials, so that there is no diffusion flux into or out of the solder. As atoms migrate through the solder, they create a state of compression near the upper boundary and tension near the lower boundary.

The boundary conditions are such that atoms do not diffuse out of the solder and into the bounding materials. Thus, the diffusion flux vanishes at the faces of the film, or

$$
\frac{d \tilde{\sigma}_{11}\left(\tilde{x}_{3}= \pm 1 / 2\right)}{d \tilde{x}_{3}}=-\frac{3 F_{3} H}{2 \Omega \sigma_{0}} .
$$

From Eq. (20), two clear limits exist. For $\Lambda / H \gg 1$, the second term is negligible and the stress field will be linear in $\tilde{x}_{3}$, namely $\tilde{\sigma}_{11}\left(\tilde{x}_{3}\right)=-\left[3 F_{3} H /\left(2 \Omega \sigma_{0}\right)\right] \tilde{x}_{3}$. This limit is consistent with the seminal analysis of Blech, in which the stress is distributed linearly along the length of the material. ${ }^{4}$

For $\Lambda / H \ll 1$, it seems tempting to entirely drop the first term in Eq. (20). However, this would result in $\tilde{\sigma}_{11}=0$ everywhere and would not satisfy the boundary conditions in Eq. (21). Instead, we have a boundary-layer effect, in which the stress in nearly zero everywhere through the thickness of the film, but deviates rapidly from zero near the boundaries to satisfy the boundary conditions. In Eq. (20), let $u$ $=\frac{d \tilde{\sigma}_{11}}{d \tilde{x}_{3}} \quad$ so $\quad$ that $\quad-\frac{4}{9}\left(\frac{\Lambda}{H}\right)^{2} \frac{d u}{d \tilde{x}_{3}}+\tilde{\sigma}_{11}^{n}=-\frac{4}{9}\left(\frac{\Lambda}{H}\right)^{2} \frac{d u}{d \tilde{\sigma}_{11}} \frac{d \tilde{\sigma}_{11}}{d \tilde{x}_{3}}+\tilde{\sigma}_{11}^{n}$ $=-\frac{4}{9}\left(\frac{\Lambda}{H}\right)^{2} \frac{d u}{d \tilde{\sigma}_{11}} u+\tilde{\sigma}_{11}^{n}=0$. Separating the variables and integrating results in $\left(\frac{d \tilde{\sigma}_{11}}{d \tilde{x}_{3}}\right)^{2}=\frac{9}{2(n+1)(\Lambda / H)^{2}} \tilde{\sigma}_{11}^{n+1}+C$, where $C$ is an integration constant. As previously argued, for $\Lambda / H \ll 1$, the stress is essentially zero over most of the domain. Thus, $\tilde{\sigma}_{11}\left(\tilde{x}_{3}=0\right) \approx 0$ and $d \tilde{\sigma}_{11} / d \tilde{x}_{3}\left(\tilde{x}_{3}=0\right) \approx 0$ so that $C \approx 0$. We now have

$$
\left(\frac{d \tilde{\sigma}_{11}}{d \tilde{x}_{3}}\right)^{2}=\frac{9}{2(n+1)(\Lambda / H)^{2}} \tilde{\sigma}_{11}^{n+1} .
$$

The maximum tensile stress will be located at the boundary, $\tilde{x}_{3}=-1 / 2$. Substituting the boundary condition in Eq. (21) into Eq. (22) gives

$$
\tilde{\sigma}_{\max }=\left[\frac{\sqrt{n+1} F_{3}}{\sqrt{2} \Omega \sigma_{0}} \Lambda\right]^{2 /(n+1)} .
$$

Hence, for a power-law creep material with $\Lambda / H \ll 1$, the maximum stress in the film scales as $\Lambda^{2 /(n+1)}$ and is independent of the thickness $H$. 
To get a better sense of how stress varies through the thickness, Eq. (20) subject to the boundary conditions in Eq. (21) was solved using the finite-element software COMSOL Multiphysics. In this software, the 1D general form in the PDE (partial differential equation) modes was selected to perform the analysis. We have used $n=3$ and $F_{3} H / \Omega \sigma_{0}=86.9$, which are representative values for a $\mathrm{SnAgCu}$ solder during operation (with $j=10^{8} \mathrm{~A} / \mathrm{m}^{2}$, $\left.T=100{ }^{\circ} \mathrm{C}, H=50 \mu \mathrm{m}\right) .{ }^{12}$ The stress distribution through the film thickness is plotted in Fig. 5 as a function of $\Lambda / H$. The stress is zero at the middle of the film, is tensile on one side, and is compressive on the other. We can clearly see a transition from the boundary layer regime to the Blech regime (linear stress distribution) as $\Lambda / H$ increases. Also from Fig. 5 , we observe that a large $\Lambda / H$ or fast diffusion relative to creep results in a relatively large stress. Likewise, slow diffusion relative to creep results in a relatively small stress. We can interpret this observation in the following way: if atomic diffusion is fast relative to creep, a large stress is built up before it can be relaxed by deformation (creep). Conversely, if creep is fast relative to diffusion, the material is liquid-like and can deform very rapidly to prevent large stress build-up.

\section{VOID FORMATION IN SOLDER BUMPS}

It is commonly suggested that voids are formed in the solder by supersaturation of vacancies at the cathode. ${ }^{24}$ The idea is that atoms diffuse by a vacancy mechanism in the direction of the electron flow; hence, vacancies migrate in the direction opposite to atomic diffusion. When enough vacancies accumulate near the cathode, they can condense to form a void, as shown in Fig. 2(a). The void can grow as additional vacancies are supplied to it. Once the voids propagate across the length of the contact, the bump fails. This physical picture is adopted by most researchers in the area.

The above picture, however, is inconsistent with the following consideration: A solder bump has numerous sources and sinks of vacancies spread throughout it, such as grain boundaries and dislocations. As noted by Balluffi, these sources and sinks tend to keep vacancy concentrations at equilibrium levels, as illustrated in Fig. 2(b). ${ }^{19}$ Hence, vacancy

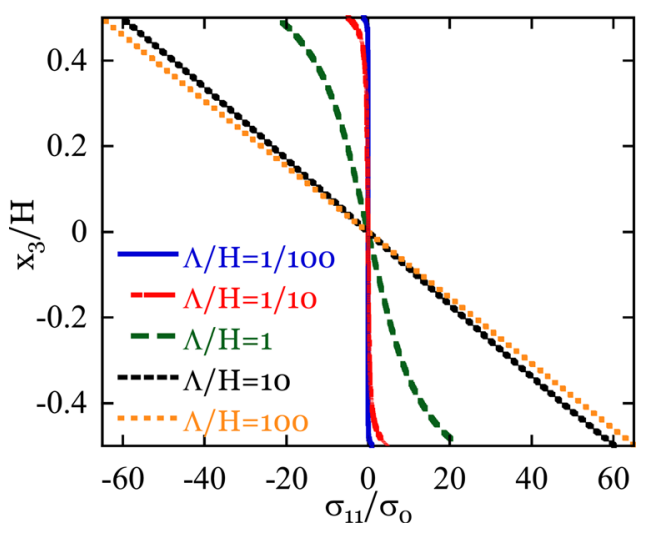

FIG. 5. (Color online) Stress distribution through the film thickness as a function of characteristic length $\Lambda$ for a material with power law creep. The stress distribution changes from a boundary layer-type distribution to a linear one as $\Lambda / H$ increases. Also, the maximum stress increases as $\Lambda / H$ increases. supersaturation is unlikely. Instead, we adopt an alternative physical picture for the formation of voids. In our picture, conduction electrons motivate atoms in the solder to diffuse, which can result in flux divergence. Under constraint, this flux divergence creates stress in the solder. Stress acts on the initial flaws in the material that were created during the manufacturing process. According to the Laplace equation, these flaws will grow, provided

$$
\sigma>\frac{2 \gamma}{a}
$$

where $\gamma$ is the surface tension and $a$ is the initial flaw radius, as in Fig. 6. We assume that the material will fail (have a big change in resistance) when these initial flaws grow. Conversely, the material will be immortal as long as the stress everywhere in the material is less than the critical stress, $\sigma_{c}=2 \gamma / a$. A similar physical picture has long been used in analyzing the formation of voids during tensile creep or the removal of pores during sintering. ${ }^{25}$

\section{ANALYSIS USING PB-FREE SOLDER DATA}

We now desire to perform a similar analysis of an actual solder system. Under a uniaxial tensile test, a double power law form commonly is found to represent the creep behavior in a solder. For instance, Wiese gives the relation for $\mathrm{SnAg}_{4} \mathrm{Cu}_{0.5}$ as

$$
\dot{\varepsilon}=A_{1} D_{1}\left(\frac{\sigma}{\sigma_{0}}\right)^{3}+A_{2} D_{2}\left(\frac{\sigma}{\sigma_{0}}\right)^{12}
$$

where $\dot{\varepsilon}$ is the creep rate in the loading direction, $\sigma_{0}=1 \mathrm{MPa}$ is a reference stress, $A_{1}=4 \times 10^{-7} / \mathrm{sec}, A_{2}=1 \times 10^{-12}$ /sec, $\quad D_{1}=\exp (-3223 / T), \quad$ and $\quad D_{2}=\exp (-7348 / T){ }^{26}$ The first term on the right hand side of Eq. (25) corresponds to the creep behavior in the low-stress regime, where dislocation climb processes dominate the deformation behavior. ${ }^{26}$ The second term corresponds to the creep behavior in the high-stress regime, where combined glide/climb processes dominate. ${ }^{26}$ Other parameters needed for the simulation include: $Z=18,{ }^{12} e=1.602 \times 10^{-19} \mathrm{C}, \quad \rho_{100^{\circ} \mathrm{C}}=1.48 \times$ $10^{-7} \mathrm{Ohm}-\mathrm{m},{ }^{27} \Omega_{S n}=2.705 \times 10^{-29} \mathrm{~m}^{3}$, and $D=1.07$ $\times 10^{-3} \exp (-105000 / R T)\left(\mathrm{m}^{2} / \mathrm{s}\right) .{ }^{28}$ It is worthwhile to note that the diffusivity given is the self-diffusivity of Sn. This value was used because the solder bumps of interest are

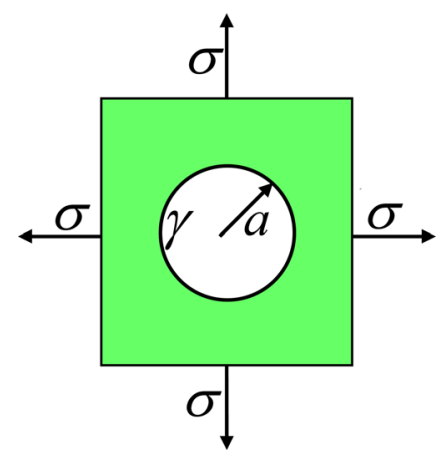

FIG. 6. (Color online) For voids to grow, we have the condition $\sigma>2 \gamma / a$, where $\gamma$ is the surface tension and $a$ is the initial flaw radius. 
composed of primarily $\mathrm{Sn}$ and because $\mathrm{Sn}$ is the dominate diffusing species in an important mode of electromigration failure. ${ }^{29}$ Also, the diffusivity used in the simulation is for diffusion along the a-axis (body-centered tetragonal crystal structure). The diffusivity along the c-axis is comparable, with an activation energy of $107 \mathrm{~kJ} / \mathrm{mol}^{28}$

With these data, the characteristic length $\Lambda$ is plotted as a function of temperature in Fig. 7. From this figure, we see that, for the above data, the characteristic length $\Lambda$ increases as the temperature increases. This occurs because the diffusivity increases more rapidly with temperature than the creep rate does. Also, in the plotted temperature range, the characteristic length is in the micron scale, which is roughly comparable to the size of a typical solder bump $(\sim 50 \mu \mathrm{m})$.

For the constitutive law in Eq. (25), the governing ordinary differential equation is

$$
-\frac{2 D \Omega}{9 k T} \frac{d^{2} \sigma_{11}}{d x_{3}^{2}}+\frac{\sigma_{11}}{2 \sigma_{0}}\left[A_{1} D_{1}\left(\frac{\left|\sigma_{11}\right|}{\sigma_{0}}\right)^{2}+A_{2} D_{2}\left(\frac{\left|\sigma_{11}\right|}{\sigma_{0}}\right)^{11}\right]=0 .
$$

The boundary conditions are

$$
\frac{d \sigma_{11}\left(x_{3}= \pm H / 2\right)}{d x_{3}}=-\frac{3 F_{3}}{2 \Omega} .
$$

As mentioned in Sec. V, we hypothesize that a critical stress exists, above which voids will grow. This critical stress is given by the Laplace equation: $\sigma_{c}=2 \gamma / a$. For $\mathrm{Sn}$, $\gamma \approx 0.5 \mathrm{~N} / \mathrm{m}^{30}$ and assuming that the initial flaw has a radius of $100 \mathrm{~nm}$, a representative value for this critical stress is $\sigma_{\text {critical }} \approx 10 \mathrm{MPa}$. It should be noted that this is a simple model to estimate the critical stress. Alternatively, the creep strengths of the material may be used if these data are available. For instance, the creep strength of a $\operatorname{SnAg}_{3.5}$ solder at $100{ }^{\circ} \mathrm{C}$ is about $5 \mathrm{MPa} .{ }^{12}$ This value is comparable to the value estimated from the Laplace equation. The main point for the purposes of this paper is that a critical stress exists and is a constant on the order of $10 \mathrm{MPa}$. This critical stress depends on material properties of the solder and on the manufacturing process.

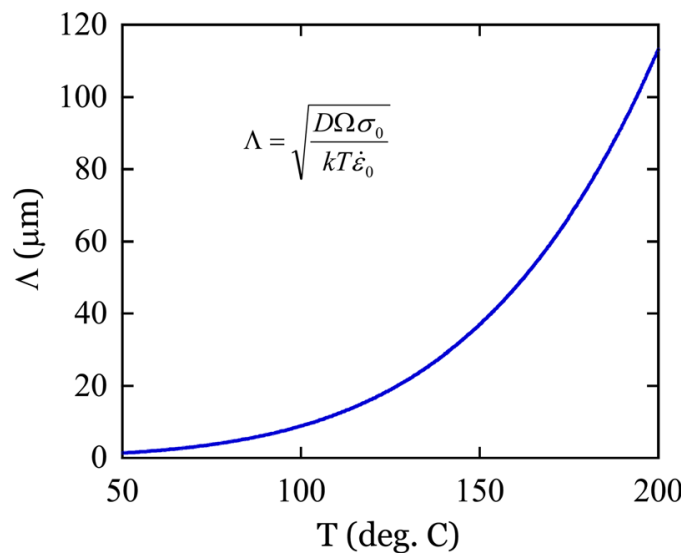

FIG. 7. (Color online) Characteristic length as a function of temperature. For $\mathrm{SnAg}_{4} \mathrm{Cu}_{0.5}$ with constitutive law in Eq. (25), $\Lambda$ increases with $T$, meaning diffusion increases more rapidly than creep does as the temperature increases.
We would like to calculate the current density necessary to reach this critical stress. For each film thickness and temperature, the current density (from $F_{i}=Z e \rho j_{i}$ ) was varied in COMSOL until a maximum stress of $\sigma_{\max }=\sigma_{c}=10 \mathrm{MPa}$ was achieved and this current density was recorded as the critical current density $j_{c}$. The critical current density as a function of thickness and temperature is plotted in Fig. 8. From this figure, for a given thickness, the critical current density decreases as the temperature increases. The explanation for this observation can be ascertained by examining Fig. 7; the characteristic length $\Lambda$ increases as the temperature increases. As previously discussed, for a given current density, an increase in $\Lambda$ results in an increase in $\sigma_{\max }$. A larger maximum stress for each current density means it requires a smaller current density to reach $\sigma_{c}$. Thus, the critical current density will decrease as temperature increases.

It is also interesting to note the functional form of $j_{c}(T)$ when $\Lambda / H \ll 1$. Although $\mathrm{SnAg}_{4} \mathrm{Cu}_{0.5}$, in general, obeys a double power law relation given by Eq. (25), for the stress levels and temperatures of interest, the first term usually is much larger than the second. Thus, it can be approximated by single power-law creep, as analyzed in Sec. IV, and we can rewrite Eq. (23) as

$$
\sigma_{c}=\left[\frac{\sqrt{n+1}\left(Z e \rho j_{c}\right)}{\sqrt{2} \Omega \sigma_{0}}\right]^{2 /(n+1)} \Lambda^{2 /(n+1)}
$$

and therefore

$$
j_{c} \propto 1 / \Lambda
$$

Also, since $\Lambda$ is given by $\Lambda=\sqrt{\frac{D \Omega \sigma_{0}}{k T \dot{\varepsilon}_{0}}}$ $=\sqrt{\frac{\Omega}{k T} D_{0} \exp \left(-\frac{Q_{D}}{k T}\right) C_{0} \exp \left(\frac{Q_{c}}{k T}\right)}$, one obtains $j_{c} \propto \sqrt{k T} \exp$ $\left(\frac{Q_{D}-Q_{C}}{2 k T}\right)$. We can further simplify this relationship by noticing that the square root term is quite weak. For instance, for an increase from $100{ }^{\circ} \mathrm{C}$ to $200^{\circ} \mathrm{C}, j_{c}$ will increase only by $13 \%$, due to the contribution from the $\sqrt{k T}$ term. The dependence on the exponential term is much stronger. Thus, we approximately have

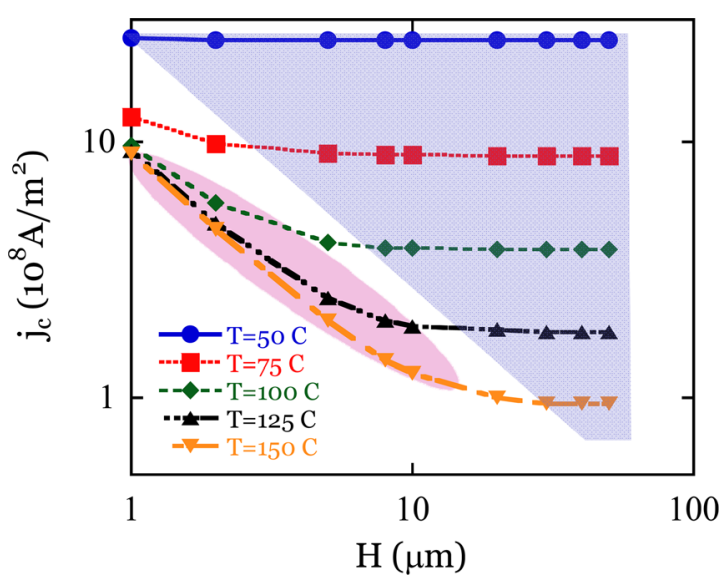

FIG. 8. (Color online) Critical current density as a function of thickness and temperature for $\mathrm{SnAg}_{4} \mathrm{Cu}_{0.5}$. The bottom left region indicates the Blech regime, and the top right region represents the boundary-layer regime. 


$$
j_{c} \propto \exp \left(\frac{Q_{D}-Q_{C}}{2 k T}\right)
$$

Thus, in the boundary layer regime, we expect an exponential dependence of the critical current density on temperature. Such a dependence cannot easily be explained in terms of Blech's analysis. In his analysis, he attributed this dependence to the increase in flow stress, i.e., a change in the maximum compressive/tensile stresses the material can withstand. Albeit a possible explanation, our theory has a more straightforward explanation, as given in the derivation above. Specifically, for a material in the boundary layer regime, the critical current density scales with the reciprocal of the characteristic length, $j_{c} \propto 1 / \Lambda$. The characteristic length depends on the diffusivity and creep law of the material, which exhibit Arrhenius-type relations. Consequently, the critical current density scales exponentially with temperature, as given in Eq. (30).

The critical product $j_{c} H$, as recognized by Blech, is plotted as a function of the thickness $H$ in Fig. 9. In his analysis, Blech claims this product should be a constant, above which electromigration damage will occur. ${ }^{4}$ The product should be independent of the size. This figure demonstrates that, for a given temperature, there is a transition from the Blech regime to the boundary-layer regime as the film thickness increases. Specifically, between 100 and $150^{\circ} \mathrm{C}$, using experimentally determined solder properties, this transition occurs between $1-10 \mu \mathrm{m}$. This means that, according to the data and the simulation, a solder larger than $10 \mu \mathrm{m}$ is actually in the boundary layer regime during operation. Thus, it appears that this boundary layer effect may be important in proper analysis of the stress distribution in $\mathrm{Pb}$-free solder bumps.

\section{COMPARISON WITH EXPERIMENTAL RESULTS}

Comparison with experiments is difficult, due to the lack of data for critical current densities in actual solder bumps. However, we can still make some comparisons to solder materials with slightly different geometries. For instance, Hsu et $a l .{ }^{31}$ measured the critical current density as a function of temperature for $350-\mu \mathrm{m}$-long Blech-type $\mathrm{SnAg}_{3.8} \mathrm{Cu}_{0.7}$ solder stripes. This material is in the boundary layer regime for the testing conditions, so we expect $\ln \left(j_{c}\right) \propto 1 / T$, as given in

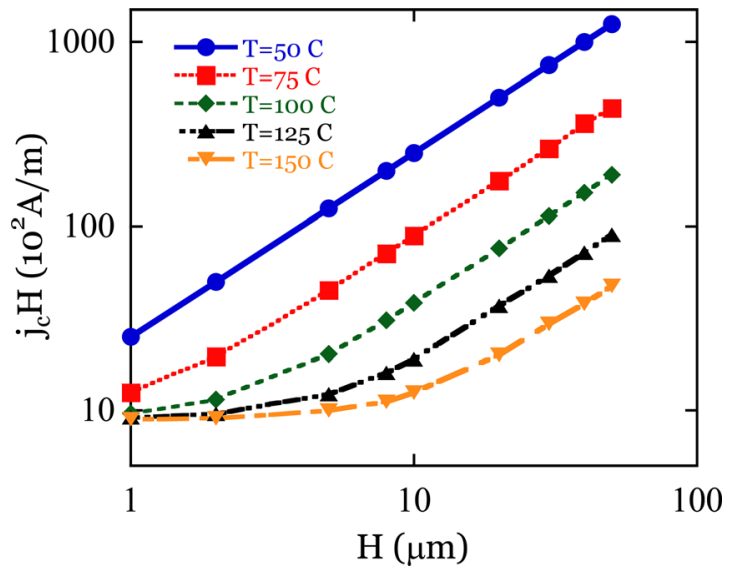

FIG. 9. (Color online) Critical product as a function of film thickness and temperature.
Eq. (30). A comparison of the simulated results (for a $350 \mu \mathrm{m}$ $\mathrm{SnAg}_{3.8} \mathrm{Cu}_{0.7}$ solder stripe) and the experimental data is given in Fig. 10. The apparent activation energy, $Q$, found from the simulation is $0.385 \mathrm{eV}$. The experimental data also shows an exponential dependence with $\mathrm{Q}=0.333 \mathrm{eV}$, comparable to the predicted value.

As another example, Yoon et al..$^{32}$ examined the dependence of the threshold current density on line length. In these experiments, five $\mathrm{SnPb}$ solder lines were tested with lengths ranging between 100 and $1000 \mu \mathrm{m}$ at a temperature of $140{ }^{\circ} \mathrm{C}$. Their experiments found that the critical current density is a constant independent of line length. Using the creep and law and diffusivity from Siewert $e t$ al $^{12}$, the characteristic length of $\mathrm{SnPb}$ is found to be $\Lambda=0.199 \mu \mathrm{m}$ at $140^{\circ} \mathrm{C}$. Thus, $\Lambda / H \ll 1$ for these experiments and we predict the $\mathrm{SnPb}$ solder to be well within the boundary layer regime. As was suggested in Sec. IV, for a solder in the boundary layer regime, we predict the critical current density to be independent of size, as was found in the experiments.

\section{3D SOLDER VERSUS THIN FILM}

In Secs. IV-VII, we have approximated the solder bump as a thin film. Initially, we desired to solve the full set of 3D governing equations, Eqs. (17) and (18), using the finite element software COMSOL Multiphysics. However, it was found that the creep law for the solder is highly nonlinear, leading to some convergence and/or memory problems. We then realized that we should be able to approximate the solder bump as a thin film, making the problem 1D. A 1D problem has a drastically reduced number of degrees of freedom, which allowed for a much finer mesh and ultimately for convergence in COMSOL.

However, we need to justify this approximation of the solder bump being represented as a thin film. To do so, we implemented Eqs. (17) and (18) in COMSOL using the general form of the PDE modes module. However, the constitutive law used was a linear relation, namely $d_{i j}^{C}=s_{i j} / 2 \eta$, where $\eta$ is the viscosity of the material. This linear problem is much easier to solve with the finite element software. The boundary conditions used are no flux through any surfaces,

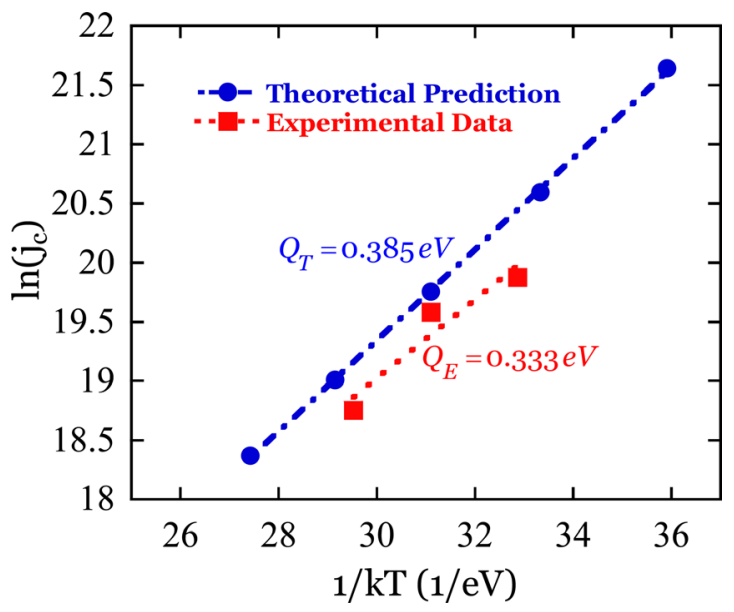

FIG. 10. (Color online) Critical current density as a function of temperature. The experimental data is for a $350 \mu \mathrm{m} \mathrm{SnAg}{ }_{3.8} \mathrm{Cu}_{0.7}$ solder stripe. 


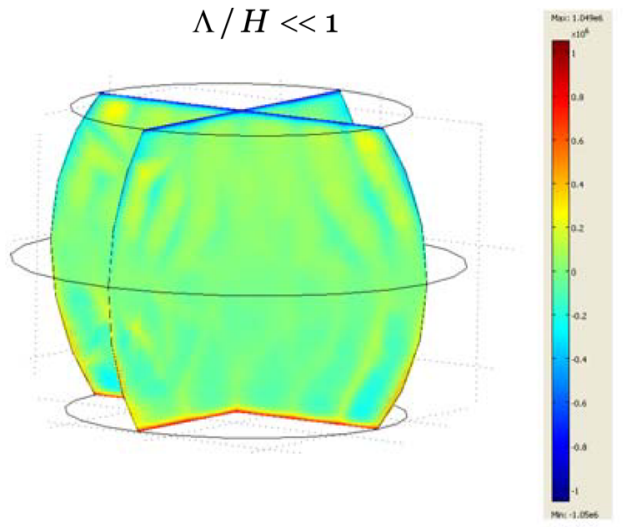

traction-free lateral surfaces, and no marker velocity on the upper and lower boundaries,

$$
\begin{aligned}
& J_{i} n_{i}=0 \quad \text { all surfaces } \\
& t_{i}=\sigma_{i j} n_{j}=0 \quad \text { lateral surfaces } \\
& v_{i}=0 \quad \text { top } / \text { bottom surfaces. }
\end{aligned}
$$

Typical stress distributions resulting from the simulation are shown in Fig. 11.

Physically, differences between the 3D model and the thin film one will arise due to stress relaxation via creep on the lateral surfaces of the 3D model. Such relaxation cannot occur in the 1D model, since the material is constrained from deforming laterally. However, for $\Lambda / H \ll 1$, we expect the stress to be confined to thin layers near the upper and lower boundaries. Since we also have a no marker velocity boundary condition at these boundaries, in these layers, we roughly have no lateral deformation. Thus, in the region where stress exists, the material is constrained laterally, suggesting that the thin film approximation is appropriate. Likewise, for $\Lambda / H \gg 1$, creep is very slow compared to diffusion. Thus, stress relaxation via creep is negligible, and the thin film approximation seems valid. The comparisons given in Fig. 12 confirm these ideas. From this figure, we can see that, in these two limiting cases, $\Lambda / H \ll 1$ and $\Lambda / H \gg 1$, the $3 \mathrm{D}$ and $1 \mathrm{D}$ simulations agree quite well. Thus, these physical justifications and simulations suggest that the approximation of the solder bump as a thin film is valid for the two limiting cases: $\Lambda / H \ll 1$ and $\Lambda / H \gg 1$.

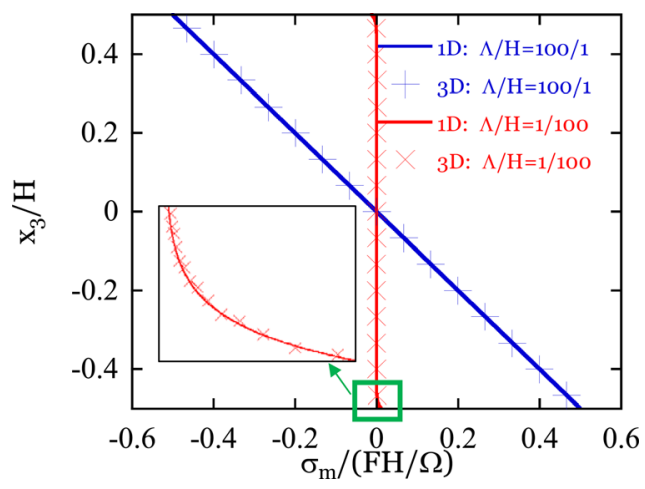

FIG. 12. (Color online) Comparison of stress in 3D and 1D simulations plotted through the thickness. A close-up view of the boxed region is given in the bottom left corner of the plot.
$\Lambda / H \gg>1$

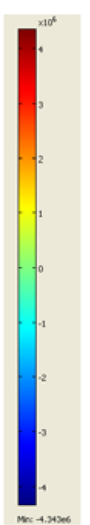

FIG. 11. (Color online) Typical stress distributions for linear creep using the 3D model in COMSOL.

\section{CONCLUSIONS}

To determine the stress field in a solder bump, we have presented a theory that couples creep and electromigration. This theory results in governing Eqs. (17) and (18), a set of coupled PDE's that allow for the calculation of the stress and deformation fields. From these equations, an intrinsic length emerges, Eq. (19), which characterizes the relative rates of creep and diffusion. When diffusion is slow relative to creep, we find the stress is relatively small and localized to the boundary of the solder. We suggest that, when stress exceeds a threshold value, voids will form in the solder. Using Pbfree solder data, we discover that the effects of creep are significant and must be taken into account for proper analysis of electromigration-induced failure in solder bumps. Comparisons with experiments demonstrate general agreement with the theory. To further augment the content of this theory, we need more experimental data on electromigration failure in $\mathrm{Pb}$-free solders. Specifically, it would be useful to have a systematic study measuring critical current density for various solder sizes and operating temperatures.

\section{ACKNOWLEDGMENTS}

The work at Harvard University is supported by a contract from Intel Corp. M.P. acknowledges government support under and awarded by DoD, Air Force Office of Scientific Research, National Defense Science and Engineering Graduate (NDSEG) Fellowship, 32 CFR 168 a.

${ }^{1}$ K. N. Tu, J. Appl. Phys. 94(9), 5451 (2003).

${ }^{2}$ E. C. C. Yeh, W. J. Choi, K. N. Tu, P. Elenius, and H. Balkan, Appl. Phys. Lett. 80(4), 580 (2002).

${ }^{3}$ I. A. Blech, J. Appl. Phys. 47(4), 1203 (1976).

${ }^{4}$ I. A. Blech and C. Herring, Appl. Phys. Lett. 29(3), 131 (1976).

${ }^{5}$ M. A. Korhonen, P. Borgesen, K. N. Tu, and C. Y. Li, J. Appl. Phys. 73(8), 3790 (1993).

${ }^{6}$ R. G. Filippi, G. A. Biery, and R. A. Wachnik, J. Appl. Phys. 78(6), 3756 (1995).

${ }^{7}$ Z. Suo, Acta Mater. 46(11), 3725 (1998).

${ }^{8}$ Y. J. Park, V. K. Andleigh, and C. V. Thompson, J. Appl. Phys. 85(7), 3546 (1999).

${ }^{9}$ R. Rosenberg, D. C. Edelstein, C. K. Hu, and K. P. Rodbell, Annu. Rev. Mater. Sci. 30, 229 (2000).

${ }^{10}$ E. T. Ogawa, K. D. Lee, V. A. Blaschke, and P. S. Ho, IEEE Trans. Reliab. 51(4), 403 (2002).

${ }^{11}$ J. He, Z. Suo, T. N. Marieb, and J. A. Maiz, Appl. Phys. Lett. 85(20), 4639 (2004).

${ }^{12}$ T. Siewert, S. Liu, D. Smith, and J. C. Madeni, Database for Solder Properties with Emphasis on New Lead-Free Solders, Properties of Lead-Free 
Solders, Release 4.0, National Institute for Standards and Technology and Colorado School of Mines, Golden, CO 80401, 2002.

${ }^{13}$ D. Tribula, D. Grivas, and J. W. Morris, J. Electron. Mater. 17(5), 387 (1988).

${ }^{14}$ Z. Zhang, Z. G. Suo, and J. He, J. Appl. Phys. 98(7), 074501 (2005).

${ }^{15}$ Z. Suo, ASME Trans. J. Appl. Mech. 71(5), 646 (2004).

${ }^{16}$ L. S. Darken, Trans. Am. Inst. Min. Metall. Eng. 175, 184 (1948).

${ }^{17}$ T. Y. Lee, K. N. Tu, and D. R. Frear, J. Appl. Phys. 90(9), 4502 (2001).

${ }^{18}$ L. H. Xu, J. H. L. Pang, and K. N. Tu, Appl. Phys. Lett. 89(22), 221909 (2006).

${ }^{19}$ R. W. Balluffi, Acta Metall. 2(2), 194 (1954).

${ }^{20}$ R. W. Balluffi and L. L. Seigle, Acta Metall. 3(2), 170 (1955).

${ }^{21}$ I. Daruka, I. A. Szabo, D. L. Beke, C. S. Cserhati, A. Kodentsov, and F. J. J. van Loo, Acta Mater. 44(12), 4981 (1996).

${ }^{22}$ Z. Suo, D. V. Kubair, A. G. Evans, D. R. Clarke, and V. K. Tolpygo, Acta Mater. 51(4), 959 (2003).
${ }^{23}$ H. B. Huntington and A. R. Grone, J. Phys. Chem. Solids 20(1-2), 76 (1961).

${ }^{24}$ K. Zeng and K. N. Tu, Mater. Sci. Eng. R. 38(2), 55 (2002).

${ }^{25}$ T. J. Chuang, K. I. Kagawa, J. R. Rice, and L. B. Sills, Acta Metall. 27(3), 265 (1979).

${ }^{26}$ S. Wiese and K. J. Wolter, Microelectron. Reliab. 44(12), 1923 (2004).

${ }^{27}$ T. Y. Lee, K. N. Tu, S. M. Kuo, and D. R. Frear, J. Appl. Phys. 89(6), 3189 (2001)

${ }^{28}$ C. Coston and N. H. Nachtrieb, J. Phys. Chem. 68(8), 2219 (1964).

${ }^{29}$ M. H. Lu, D. Y. Shih, P. Lauro, and C. Goldsmith, Appl. Phys. Lett. 94(1), 011912 (2009).

${ }^{30}$ I. Kaban, S. Mhiaoui, W. Hoyer, and J. G. Gasser, J. Phys.: Condens. Matter 17(50), 7867 (2005).

${ }^{31}$ Y. C. Hsu, C. K. Chou, P. C. Liu, C. Chen, D. J. Yao, T. Chou, and K. N. Tu, J. Appl. Phys. 98(3), 033523 (2005).

${ }^{32}$ M. S. Yoon, M. K. Ko, B. N. Kim, B. J. Kim, Y. B. Park, and Y. C. Joo, J. Appl. Phys. 103(7), 073701 (2008). 